\title{
A 16-Channel 8-Parameter Waveform Electrotactile Stimulation System
}

\author{
Kurt A. Kaczmarek, Student Member, IEEE, Kevin M. Kramer, Student Member, IEEE, \\ John G. Webster, Fellow, IEEE, and Robert G. Radwin, Member, IEEE
}

\begin{abstract}
We have developed a general-purpose electrotactile (electrocutaneous) stimulation system as a research tool for studying psychophysiological performance associated with various stimulation waveforms. An experimenter-defined command file specifies the stimulation current and waveform of each of the 16 channels. The system provides burst onset delay of 0-20 ms, phase current of $0-50 \mathrm{~mA}$, interphase interval of 0$1000 \mu \mathrm{s}$, number of pulses per burst from 1-100, pulse repetition rate of $0.1-25 \mathrm{kHz}$, phase width of $2-1000 \mu \mathrm{s}$, and functionally-monophasic pulses (with zero dc current) or balancedbiphasic pulses (with equal positive and negative phases). The system automatically delivers the desired stimulation, prompts the subject for responses, and then logs subject responses. Key features of the system are 1) very flexible choice of bursts of pulsatile waveforms, 2) real-time control of all of the waveform parameters as mathematical functions of external analog inputs, and 3) high-performance electrode-driver circuitry.
\end{abstract}

\section{INTRODUCTION}

$\mathrm{E}^{2}$ LECTROTACTILE STIMULATION evokes tactile (touch) sensations within the skin at the location of the electrode by passing a local electric current through the skin. Sensory substitution is the use of one human sense (in this case, touch) to receive environmental information normally received by another sense (often vision or hearing). For the sense of touch, sensory substitution is the use of one area of skin to receive tactile information normally received at another location. Several papers review technology and devices for electrotactile simulation [1], [2], visual substitution [3], [4], auditory substitution [5]-[7], and other applications [2], [8]-[12].

\section{Limitations of Present Electrotactile Displays}

Insufficient Dynamic Range: A substantial limitation of present electrotactile displays is that they lack sufficient intensity dynamic range. Our normal senses of vision, hearing, and touch can mediate stimuli which we perceivè as strong or intense without being painful. Electrotactile stimulation, on the other hand, can develop an uncom-

Manuscript received March 13, 1990; revised December 17, 1990. This work was supported by the National Institutes of Health, Grant NS26328.

K. A. Kaczmarek, K. M. Kramer, and J. G. Webster are with the Department of Electrical and Computer Engineering. University of Wisconsin, Madison. WI 53706

R. G. Radwin is with the Department of Industrial Engineering, University of Wisconsin. Madison. WI 53706.

IEEE Log Number 9102473. fortable stinging quality even at moderate stimulation levels if improper stimulation waveforms or electrodes are used. The traditional measure of intensity dynamic range is the ratio of the stimulation currents required to produce sensation threshold $I_{S}$ and pain threshold $I_{P} . I_{P} / I_{S}$ typically ranges from $2-4$ for unexperienced subjects and 6-8 for experienced subjects [13]. This range is a limitation for intensity-modulated stimulation codes but not necessarily for frequency or spatially-modulated codes.

Interpretation of Unfamiliar Sensations: Electrotactile stimulation produces sensations that are often unfamiliar and not comparable to normal touch sensations. In particular, the relationship between the stimulus intensity (current) and the perceived sensation is quite different than that for normal touch. Research using this instrumentation will provide information to assist in the design of appropriate information processing schemes for electrotactile displays [1].

\section{Required Instrumentation}

To determine optimal stimulus waveforms, we require a system which independently controls all eight waveform parameters (defined below) for several channels of electrotactile stimulation. For independent channel control, the output circuit should simultaneously drive several electrodes without interchannel interaction due to output circuit multiplexing such as that used in the Tacticon auditory prosthesis [7], [11]. Finally, the system should be capable of administering predefined experiments to subjects and automatically recording their responses with minimal experimenter intervention during the session. We therefore developed this custom electrotactile stimulation system (ETSS).

\section{Waveform and Electrode Definitions}

All eight of the waveform parameters in Table I and all seven of the electrode parameters in Table II influence the electrotactile sensation. Fig. 1 shows the pulse timing relationships; Fig. 2 shows the four pulse types. Fig. 3 defines the generalized single-electrode parameters. The eight waveform parameters define a generalized stimulation waveform; proper choice of parameters can describe any rectangular electrotactile stimulation waveform previously described in the literature. 
TABLE I

Electrotactile WAVEForm Parameters

\begin{tabular}{|c|c|c|c|c|}
\hline Parameter & Symbol & ETSS Range & Resolution & Unit \\
\hline Burst onset delay & $D$ & $0-120$ & 0.002 & $\mathrm{~ms}$ \\
\hline Burst rate & $F$ & $1-1000$ & $\#$ & $\mathrm{~Hz}$ \\
\hline Phase current & 1 & $0-50$ & 0.00122 & $\mathrm{~mA}$ \\
\hline $\begin{array}{l}\text { Interphase } \\
\text { interval }\end{array}$ & IPI & $0-1000$ & 0.2 & $\mu \mathrm{s}$ \\
\hline Pulses/burst & $N P B$ & $1-100$ & 1 & pulses \\
\hline Pulse rate & $P R R$ & $0.1-25$ & $\#$ & $\mathrm{kHz}$ \\
\hline Phase width & $W$ & $2-1000$ & 0.2 & $\mu \mathrm{s}$ \\
\hline Pulse type & Type & $\mathrm{M}+, \mathrm{M}-, \mathrm{B}+, \mathrm{B}-$ & N/A & \\
\hline $\begin{array}{l}\text { Pulse repeat } \\
\text { period }\end{array}$ & $P^{*}=1 / P R R$ & $0.04-10$ & 0.002 & $\mathrm{~ms}$ \\
\hline $\begin{array}{l}\text { Time between } \\
\text { bursts }\end{array}$ & $T^{*}=1 / F$ & $1-1000$ & 0.02 & $\mathrm{~ms}$ \\
\hline
\end{tabular}

${ }^{*} P$ and $T$ are redundant parameters, being derived directly from $P R R$ and $F$, respectively.

"The resolution of $F$ and $P R R$ vary over the operating range to correspond with the resolutions of $T$ and $P$, respectively.

TABLE II

Electrotactile Electrode Parameters

\begin{tabular}{ll}
\hline \multicolumn{1}{c}{ Parameter } & Symbol \\
\hline Active electrode diameter & $D_{A}$ \\
Insulating ring diameter & $D_{,}$ \\
Dispersive electrode diameter & $D_{D}$ \\
Body location & Loc \\
Electrode separation & Sep \\
Electrode material & Mat \\
Skin preparation technique & Prep \\
\hline
\end{tabular}

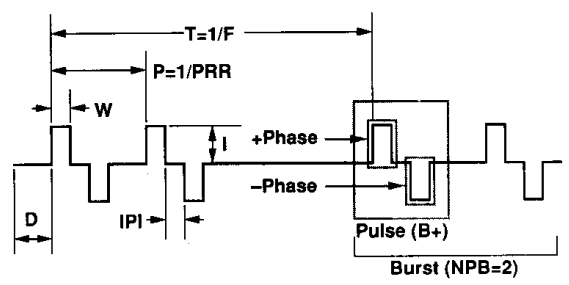

Fig. 1. Electrotactile waveform parameters: $D$, delay; $W$, width; $I P I$, interphase interval; $I$, current; $T$, time between bursts; $F$, frequency of burst repetition: $P$, period of pulse repetition; $P R R$, pulse repetition rate; $N P B$ number of pulses per burst.
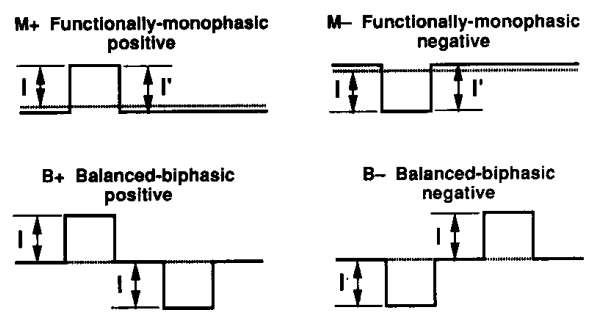

Fig. 2. Electrotactile waveform pulse types. Average current is zero for all types. The dotted line is the zero-current reference.

The literature contains inconsistent waveform terminology. Frequently, $M+$ and $M$ - waveforms as shown in Fig. 2 (with zero net de current and nonzero baseline)

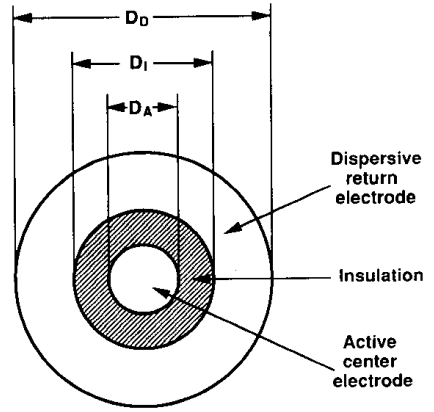

Fig. 3. In electrotactile electrodes, current enters through the active center electrode and returns through the annular dispersive return electrode. This focuses the region of highest current density to the skin region directly beneath the active electrode to provide well-localized sensations. Typical electrode dimensions are 2-10 mm for $D_{A}, 1-4 \mathrm{~mm}$ for $D_{I}-D_{A}$, and 4-100 $\mathrm{mm}$ for $D_{b}$.

are called "biphasic" because they have positive and negative parts. $B+$ and $B-$ are sometimes called "biphasic with equal positive and negative parts." A zerobaseline monophasic waveform (with a net dc current) is never used for electrotactile stimulation due to rapid skin irritation resulting from electrochemical reactions at the electrode-skin interface [11]. Therefore, we use the terms "functionally-monophasic" for $M+$ and $M-$ and "balanced-biphasic'" for $B+$ and $B$ - to avoid ambiguity.

Finally, the two phases of a balanced-biphasic waveform pulse (Fig. 1) are often called pulses (with the result that interphase interval is called "interpulse interval"). Introducing the term "phase" avoids the above ambiguity, and uniquely specifies the interpulse and interphase timing relationships.

\section{ETSS System Functions \\ Waveform Parameter Control}

Each of the 16 independently-controllable stimulation channels can deliver waveforms within the parameter limits in Table I. Parameter changes in one channel do not affect the waveform in any other channel. The "Hardware-Performance"' section elaborates on the parameter control tolerances.

All of the waveform parameters can be controlled in real time by mathematical functions of time or external analog inputs from subject-controlled knobs or sensors. Furthermore, the stimulation parameters for one experimental trial may be functions of earlier subject responses in the experiment.

\section{Automated Experiments}

An experimenter-defined command file controls the following functions:

1) Fixed waveform parameters for each channel.

2) Source of dynamic variation of waveform parameters (time or an external analog input).

3) Order of stimulus presentation (randomization available). 
4) Timing of stimulus presentation.

5) Subject instructions and prompts.

6) Output file format.

\section{HARDWARE}

Fig. 4 shows that the ETSS consists of four major parts. The waveform generator (WG) produces repetitive rectangular voltage pulse trains which the voltage-to-current converter (VIC) converts to constant-current pulses; these pulses stimulate the subject through skin electrodes. The analog system receives analog control signals from a user interface such as a potentiometer, or from force or pressure sensors, and provides signals which control the current of each of the 16 stimulation channels. The IBM personal computer (PC) directly controls the WG through the $\mathrm{PC}$ bus and through the analog system. It receives status information from the WG and receives external analog data from the analog system. Finally, the PC logs subject responses and system status information in an output file.

\section{Waveform Generator}

The WG consists of a PC bus interface (Fig. 5), 16 programmable timer circuits (Fig. 6), and a waveshaping circuit (Fig. 7). Fig. 8 shows the interconnections between Figs. 6 and 7 for the 16-channel WG. Fig. 9 shows the common 5-MHz clock for the timers.

Bus Interface: Each of the Am9513 timer circuits (Fig. 6) is controlled by two bidirectional input/output $(\mathrm{I} / \mathrm{O})$ ports. The bus interface maps each of the timers consecutively into the PC I/O space B00-B1F hex. Only one 9513 timer is accessible at a time.

Timer Selection: Advanced Micro Devices (Sunnyvale, CA) Am9513 programmable timer integrated circuits perform all of the timing functions in generating the pulsatile stimulation waveforms. They are controllable in real time by an extensive set of software commands and are easily interfaced to the PC bus. A separate timer IC for each channel controls the seven waveform timing parameters for each channel.

Timer Description: One off-chip master crystal-controlled 5-MHz clock (Fig. 9) provides the time base for all 16 IC's. Each 9513 timer IC has five independentlycontrollable counters, each with programmable clock rate and source, start/stop trigger conditions, numeric preload, and reset capability.

Counter Functions: Fig. 10(a) shows the functional counter interconnections for one channel of waveform generation. Using the waveform definitions in Table I, Fig. 10(b) shows that counters 1 and 2 produce the positive and negative phases, respectively, of the stimulation waveform. These counters function as hardware-triggered delayed-pulse one-shot generators and control phase width $W$, interphase interval $I P I$, and Type (monophasic, biphasic) waveform parameters via software commands. Counter 3 operates as a rate generator with level gating, thereby controlling the pulse-repetition rate $P R R$. While

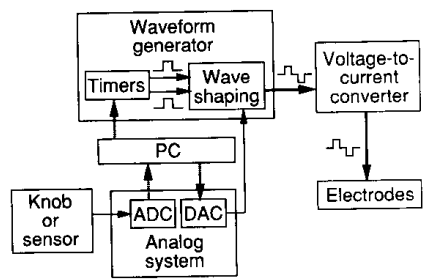

Fig. 4. Electrotactile stimulation system. All arrows to and from PC are PC bus interface connections. Solid lines are steady or slowly-varying analog signals. Heavy solid lines are pulsatile waveforms.

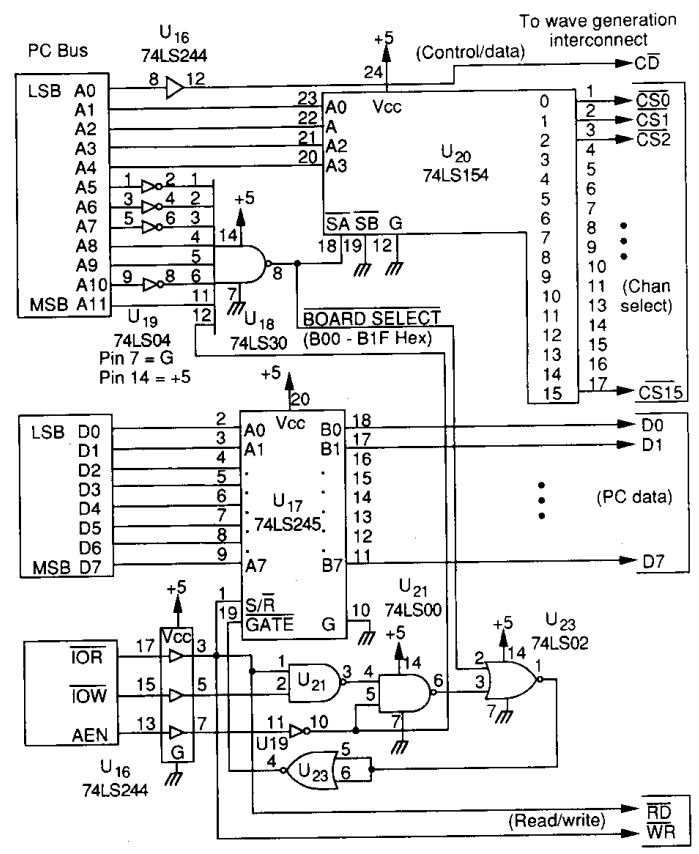

Fig. 5. The PC bus interface decodes software-generated signals which set the timers in the waveform generator.

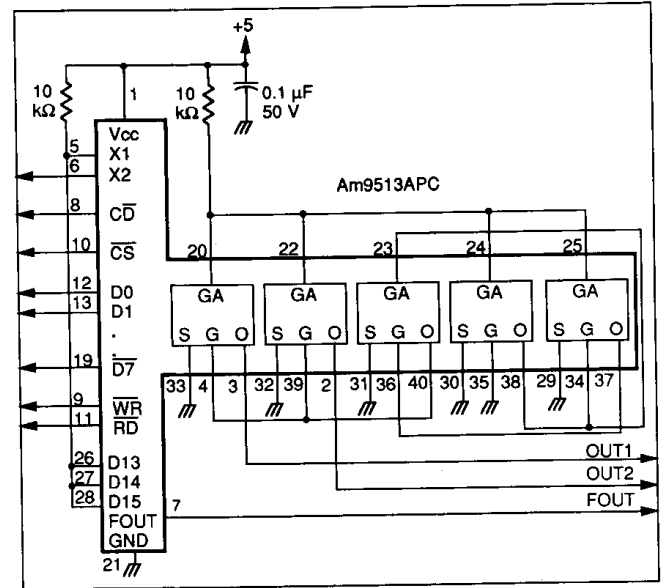

Fig. 6. One channel of programmable timers which are set to generate the waveform. 


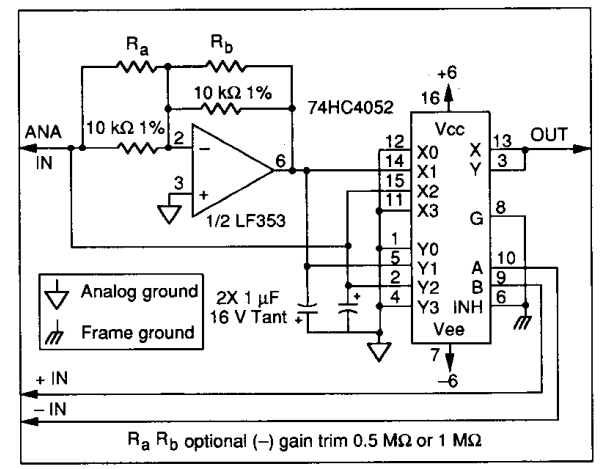

Fig. 7. One channel of waveshaping circuits.

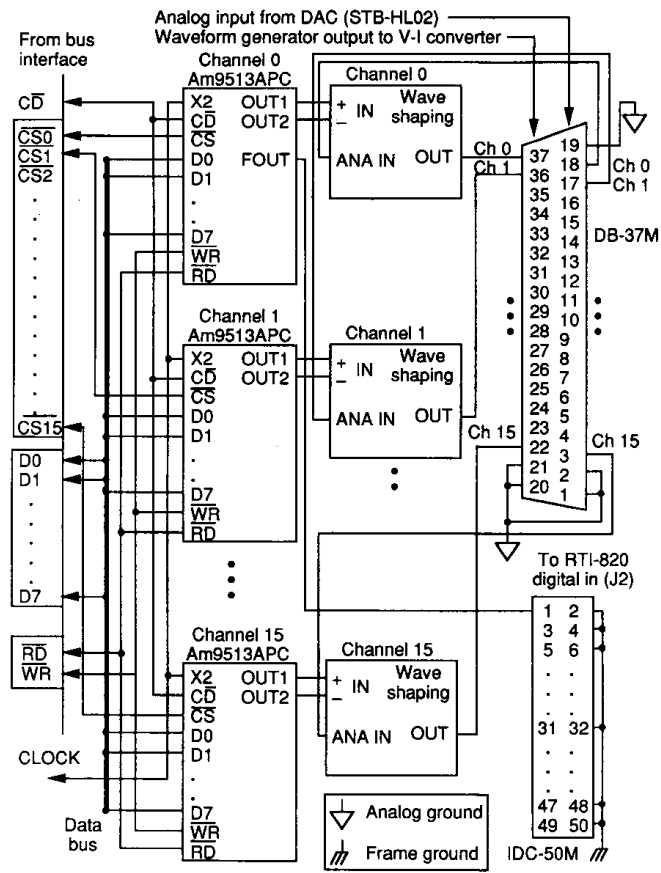

Fig. 8. Waveform generator interconnect: timer outputs and DAC analog outputs combine in the waveshaping circuit to provide waveform generator output to the voltage-to-current converter.

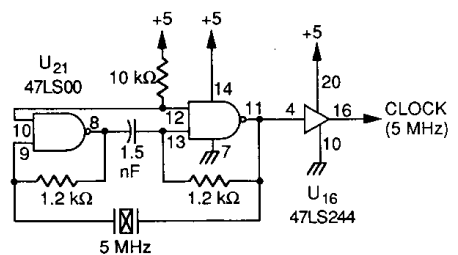

Fig. 9. Two NAND gates and a $5-\mathrm{MHz}$ crystal form the common clock for all 16 channels.

counter 3 is on, counters 1 and 2 produce pulses at a constant rate. Counter 3 may be gated off by either counter 4 or 5 . Counter 4 controls the burst-onset delay $D$ by gating counters 3 and 5 off. It is a software-triggered strobe with

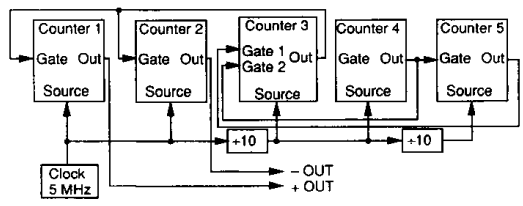

(a)

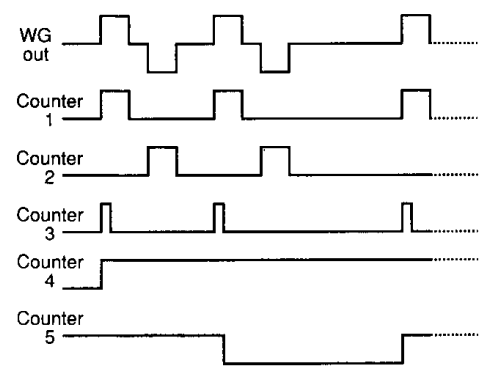

(b)

Fig. 10. (a) Some of the counter interconnections for Am9513 timer circuit are internally controlled via software. (b) Timer counters 1 and 2 control $W, I P I$, and Type waveform parameters via software commands. Counter 3 controls $P R R$, counter 4 controls $D$, and counter 5 controls $F$ and $N P B$ via software commands.

no gating. Finally, counter 5 is a variable duty cycle rate generator with level gating. While its output is high, counter 3 is active which causes counters 1 and 2 to produce pulses. If the output of counter 5 is low, counter 3 is gated off and no pulses are produced. Counter 5 controls the burst repetition frequency $F$ and the number of pulses per burst $N P B$ via software commands.

Waveshaping: Figs. 7 and 10(b) show how the output from the 9513 counters 1 and 2 control the timing of a $B+$ stimulation waveform. When the output from counter 1 is high, an analog multiplier gates the analog signal from the analog section directly to the WG output, producing the positive phase of the stimulation waveform. A high counter 2 outputs similarly produces a negative phase by gating an inverted version of the analog control signal. In all other cases the WG output is zero. By controlling counters 1 and 2 with software, the waveshaping circuit can produce all of the four pulse types: $M+, M-, B+$, and $B$-. The analog control signal sets the stimulation current.

Part Selection: The National Semiconductor 74HC4052 analog multiplexer is a single IC that can perform the conversion of counter output pulses to the desired WG output waveform. This IC has a maximal supply voltage of $\pm 7.5 \mathrm{~V}$, necessitating a separate power supply of $\pm 6 \mathrm{~V}$

Trimming of \pm Phases: Because the voltage-to-current converter (VIC) gain is typically different for positive and negative phases, adding $R_{a}$ or $R_{b}$ in Fig. 7 allows the negative phase amplitude to be trimmed upward and downward, respectively, with respect to the positive phase. For example, selecting $R_{a}=1 \mathrm{M} \Omega$ will make the negative phase amplitude approximately $1 \%$ larger than the positive phase amplitude. 
Analog Ground: To prevent a ground loop which could cause a common-mode noise signal to appear on the ground lead from the analog section to the WG, the signal ground is isolated from the PC chassis ground (Figs. 7, $8)$.

\section{Voltage-To-Current Converter-Simplified Circuit}

Fig. 11 shows a simplified form of the VIC which converts only positive voltage pulses to positive current pulses.

Current Control Loop: The voltage-to-current function is performed by the loop consisting of $U 1$ and $Q_{3}$. The high gain of $U 1$ forces $v_{E 3}$ to be very close to $v_{1}$, the input voltage. Therefore,

$$
i_{E 3}=\frac{v_{E 3}}{R_{5}}
$$

and

$$
i_{C 3}=\frac{\beta}{\beta+1} i_{E 3} \text {. }
$$

Therefore, the input voltage is converted into a current.

Current Mirror: $Q_{5}$ and $Q_{7}$ form a Wilson current mirror [14], which reflects $i_{C 3}$ to the load current $i_{L}$ and allows the load to be grounded. This also allows multiple electrodes to share a common return path. We chose a Wilson mirror for three reasons. 1) It has better matching of input and output current, 2) it is less dependent on transistor $\beta$ variations than a conventional mirror, and 3) it does not require the $\beta$ of $Q_{7}$ to be matched to $Q_{5 a}$ or $Q_{5 b}$. Therefore, we were able to use a high-voltage transistor for $Q_{7}$ as required. A conventional mirror would have required matched-pair transistors which could withstand $240 \mathrm{~V}$ and these were not readily available.

If all of the transistors in Fig. 11 have similar current gain $\beta$ and $Q_{5 a}$ and $Q_{5 b}$ are matched

$$
i_{L}=\frac{1}{1+2 /\left(\beta^{2}+\beta\right)} i_{C 3} \text {. }
$$

Static Transfer Function: Combining (1)-(3), and noting that $\beta \approx 100$ for these transistors and $R_{5}=100 \Omega$, the overall static transfer function (transconductance) for the $V-I$ converter is

$$
\frac{i_{L}}{v_{1}}=\frac{0.99}{R_{5}}=g_{m}
$$

or approximately $10 \mathrm{~mA} / \mathrm{V}$. Finally, $g_{m}$ is relatively independent of temperature, load resistance, and power supply voltage.

\section{Voltage-to-Current Converter-Full Circuit}

This section describes the additional circuitry which improves the performance and safety of the circuit in Fig. 11. Fig. 12 shows one complete channel for the VIC (less power supply, common-mode buffer, and switching).

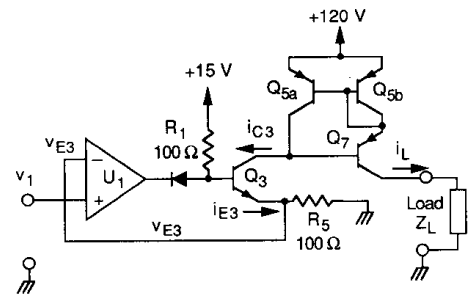

Fig. 11. Simplified voltage-to-current converter. $V_{1}$ appears across $R_{5}$. The resulting current is reflected by the current mirror $\left(Q_{5}, Q_{7}\right)$ and flows through the load.

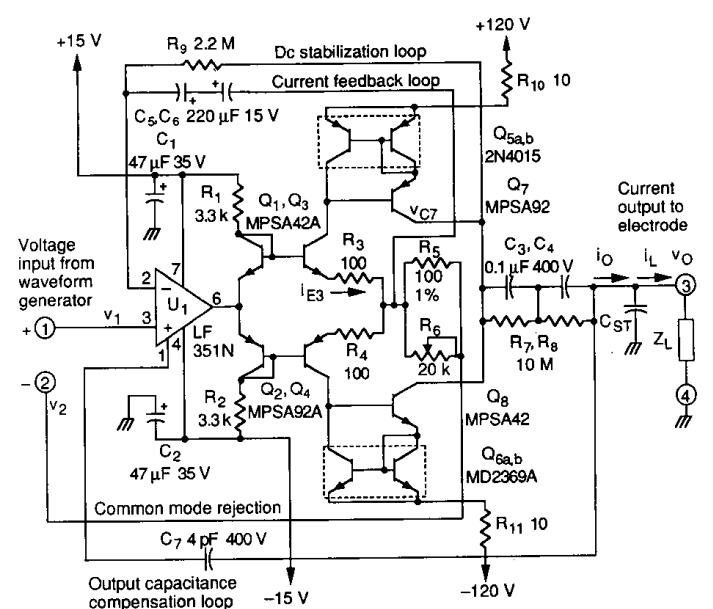

Fig. 12. Bipolar voltage-to-current converter has feedback loops to stabilize the dc operating point and compensate for output cable capacitance Some of the special part requirements are: $C_{3}, C_{4}$ are metal film polyester; $C_{7}$ is ceramic: all transistors are Motorola; $Q_{1}$ and $Q_{3}$ are thermally coupled: $Q_{2}$ and $Q_{4}$ are thermally coupled; $Q_{5}$ sub is RCA SK9115: $Q_{6}$ sub is RCA SK9114; all resistors $1 / 4 \mathrm{~W} 5 \%$ carbon. $U_{1}$ is National date code 8906 or later. Resistance in $\Omega$ unless noted; adjust $R_{6}$ for circuit transconductance $g_{m}=10 \mathrm{~mA} / \mathrm{V} . C_{\mathrm{ST}}$ is the stray capacitance from the output terminal to ground.

Differential Input: To provide rejection of commonmode noise which develops on the long input ground lead, the VIC has a differential input. The current feedback loop consisting of $C_{5}$ and $C_{6}$ causes the voltage at the junction of $R_{3}$ and $R_{5}$ to be nearly equal to $v_{1}$, so

$$
i_{E 3}=\frac{v_{1}-v_{2}}{R_{5} \| R_{6}}
$$

where

$$
R_{5} \| R_{6}=\frac{R_{5} R_{6}}{R_{5}+R_{6}} .
$$

Therefore, $i_{L}$ is dependent on $v_{1}-v_{2}$ rather than on $v_{1}$ only.

Common-Mode Buffer: A wideband, high-current buffer circuit (Fig. 13) provides the necessary current (up to 50 $\mathrm{mA}$ per channel) to drive the $(-)$ input of the VIC in Fig. 12. 


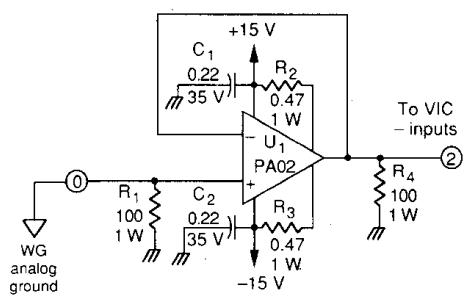

Fig. 13. A high-current buffer provides up to $50 \mathrm{~mA}$ to drive the negative input of all 16 voltage-to-current converter circuits to reduce common-mode interference. $U_{1}$ is Apex Microtechnology (Tucson, AZ) type PA02. All $R$ in $\Omega$, all $C$ in $\mu \mathrm{F}$

Decoupling and Bypassing: In Fig. $12, C_{1}$ and $C_{2}$ provide bypassing for the low-voltage supply to prevent cross-channel interference. $R_{10}$ and $R_{11}$ decouple each channel from the high-voltage supply. Without these resistors, high-frequency current transients which occur when the $Q_{5}, Q_{6}, Q_{7}$, and $Q_{8}$ switch on and off in one channel will distort the output waveform from physicallyadjacent channels. No value of bypass capacitor was sufficient to provide this decoupling without $R_{10}$ and $R_{11}$.

Rejection of $d c$ : Fig. 12 shows that the current feedback loop is capacitively-coupled. $C_{5}$ and $C_{6}$ pass stimulation pulses with widths $(W)$ up to approximately $10 \mathrm{~ms}$ with minimal distortion, effecting current control as in the simplified circuit. $R_{9}$, however, provides a dc negativefeedback path from the circuit output. The high overall circuit voltage gain forces the dc component of $v_{C 7}$ to track the dc component of $v_{1}$, which is $<0.5 \mathrm{~V}$ for any reasonable functionally-monophasic stimulation waveform (for balanced-biphasic waveforms, it is zero). The resulting net dc load current is $<25 \mathrm{nA}$ for a linear load. Because an electrode load is nonlinear [15], an additional net dc voltage source of up to $1.25 \mathrm{~V}$ (for strong stimulation by a 5.5- $\mathrm{mm}$ diameter metal electrode) can appear due to rectification of the asymmetrical functionally-monophasic waveform. The resulting maximal net dc electrode current is $(1.25 \mathrm{~V}+0.5 \mathrm{~V}) / 20 \mathrm{M} \Omega=87.5 \mathrm{nA}$.

One result of this dc rejection is that the output current pulse amplitude is lower than expected because the input waveform is dc-shifted. For functionally-monophasic pulses we must therefore modify (4):

$$
\frac{i_{L}}{v_{1}}=\frac{0.99}{R_{5}}(1-\text { DUTY })=g_{m}
$$

because $I=(1-$ DUTY $) \cdot I^{\prime}$ in Fig. 2 where DUTY $=$ $N P B \cdot W / T$ is the duty cycle for functionally-monophasic stimulation. To compensate, the software multiplies the desired functionally-monophasic current by $1 /(1-$ DUTY).

Output ac Coupling: To prevent the dangerous flow of dc current from the $120-\mathrm{V}$ supply to the subject should one of the output transistors fail, $C_{3}$ and $C_{4}$ couple the electrode to the output transistors $Q_{7}$ and $Q_{8} . R_{7}$ and $R_{8}$ serve to equally distribute any residual dc drop that may develop across these capacitors.
Bias Point Stabilization: The quiescent current through $Q_{3}$ and $Q_{4}$, and hence, $Q_{5}, Q_{6}, Q_{7}$, and $Q_{8}$, is maintained at approximately $0.5-1 \mathrm{~mA}$ by $R_{1}, R_{2}, R_{3}$, and $R_{4} \cdot Q_{1}$ and $Q_{2}$ are diode-wired and thermally-coupled to $Q_{3}$ and $Q_{4}$, respectively, to prevent temperature changes from adversely affecting the bias point.

Output Stray Capacitance Compensation: Because the output circuit is a current source, stray capacitance between the output and ground creates a first-order lag (lowpass filter), distorting the current waveform $i_{L}$ at the electrode load $Z_{L}$. The stray capacitance to ground $\left(C_{\mathrm{ST}}\right.$ in Fig. 12) is approximately $55 \mathrm{pF}$ in the output circuit, 155 $\mathrm{pF}$ in the $2-\mathrm{m}$ output cable, and $45 \mathrm{pF}$ in the electrode array. Because the resistive part of the electrode load varies more than an order of magnitude with current [15], [16], a simple lead-compensation network would not correct this effect. Instead, $C_{7}$ provides a differentiated positive-feedback path from the circuit output to its input, so that in Fig. 12

$$
\begin{aligned}
I_{L}(s) & =I_{O}(s)-s C_{\mathrm{ST}} V_{O}(s) \\
& =g_{m}\left[V_{1}(s)-V_{2}(s)+s K C_{7} V_{o}\right]-s C_{\mathrm{ST}} V_{O}(s) .
\end{aligned}
$$

where $K$ is a constant determined by the input circuit characteristics of $U_{1}$. Noting that $V_{O}(s)=I_{L}(s) Z_{L}(s)$, the overall transfer function of this circuit then becomes (assuming an otherwise ideal voltage-to-current converter)

$$
\frac{I_{L}(s)}{V_{1}(s)-V_{2}(s)}=\frac{g_{m}}{1-s Z_{L}\left(K C_{7}-C_{\mathrm{ST}}\right)} .
$$

Proper choice of $C_{7}$ will therefore cancel the effect of stray output capacitance $C_{\mathrm{ST}}$, independent of the load impedance $Z_{L}$ (see "Performance," below). The value of $C_{7}$ is dependent on the output cable length. For a 2-m output cable, $4 \mathrm{pF}$ is appropriate and is small enough to provide a nearly ideal differentiated feedback.

One of the dc-offset-correction terminals on $U_{1}$ is used as an additional input to avoid using a second op-amp as a summer. This unconventional usage requires National LF351 circuits with a date code of 8906 or later. Earlier units have a much lower "input"' resistance at pin 1 (160 $\Omega$ versus $14 \mathrm{k} \Omega$ ), preventing proper operation of this circuit.

Gain Adjustment: Since the circuit gain in (4) is slightly less than $10 \mathrm{~mA} / \mathrm{V}$ in the simplified circuit, variable $R_{6}$ is added which allows $g_{m}$ to be trimmed to 10 $\mathrm{mA} / \mathrm{V}$. This is shown in (5) and (6).

Physical Layout: Because this is a high-gain, wideband circuit with a high output impedance, stray capacitance between input and output must be minimized to prevent undesired oscillations due to capacitive coupling. While a single-channel prototype never oscillated, we experienced difficulty preventing high-frequency oscillations $(\approx 3 \mathrm{MHz})$ when two or more channels were operated in close proximity. Power-supply bypassing at each LF351 was helpful. Most of the cross-channel coupling, however, occurred in the output cable to the electrodes. A flat ribbon cable with a grounded lead between each 
pair of output leads provided sufficient interchannel isolation while avoiding the bulk and high capacitance to ground of 16 individually shielded leads. When this cable configuration connected the output circuit board to the electrode array, the oscillations disappeared.

Semiconductor Selection: $Q_{3}$ and $Q_{4}$ need to withstand $120 \mathrm{~V} ; Q_{7}$ and $Q_{8}$ need to withstand $240 \mathrm{~V}$, yet all must operate at low currents of 0.1 to $50 \mathrm{~mA}$. Otherwise their characteristics are not critical. Power consumption is low ( $<600 \mathrm{~mW}$, usually much lower) because the driving waveform duty cycle never exceeds $10 \%$. We chose $300-\mathrm{V}, 600-\mathrm{mW}$ units. Selection of $Q_{5}$ and $Q_{6}$ proved more difficult. Few matched-pair devices exist in complementary (NPN and PNP) forms with flat $\beta$ and matched characteristics from 0.1 to $50 \mathrm{~mA}$. We chose the best available and correct for their nonlinearity in software and correct for NPN-PNP mismatch in the waveform generator circuit.

Power Supplies: The VIC requires two power supplies: low-voltage $( \pm 15 \mathrm{~V})$ and high-voltage $( \pm 120 \mathrm{~V})$. The 15-V supply must be energized first and de-energized last to minimize output transients. A three-position power switch (off, standby, operate) provides this function. An optical subject-operated footswitch can also disable the $120-\mathrm{V}$ supply for emergency shutdown of stimulation.

Furthermore, a current surge which occurs upon application of the $120-\mathrm{V}$ supply requires that this supply (Acopian 120GT05D) receive ac line power before connection to the VIC circuit (also provided by the three-position power switch). Failure to follow this procedure results in automatic supply shutdown.

\section{Analog System}

We chose an analog data acquisition and control system from Analog Devices (Norwood, MA) because of its flexibility and software support. Its model RTI-820 circuit board which resides in the PC contains one analog-to-digital converter (ADC) and one digital-to-analog converter (DAC), in addition to three 8-bit parallel ports. Four peripheral model STB-HL02 interface boards containing analog multiplexers provide a total of 64 channels of analog input to the PC and 16 channels of analog output from the $P C$. The analog inputs receive signals from subject-controlled potentiometers or from force to pressure sensors. The analog output signals control the current of the 16 stimulation channels.

\section{IBM Personal Computer}

An IBM PC controls the ETSS. The PC is equipped with 640 kbytes of RAM, one 360-kbyte floppy disk drive, a 10-Mbyte hard disk drive, an 8087 numeric coprocessor, a serial port to communicate with a Diablo 630 printer, and a real-time clock (MI0-100 Multi-I/O card). Although not required for ETSS operation, the Microway (Kingston, MA) Number Smasher/ECM and Motherboard Accelerator speed up data logging and waveform updating on the system by 1) substituting an 8086 proces- sor for the PC's 8088 and 2) increasing the PC clock speed from 4.77 to $12 \mathrm{MHz}$. An upgraded $(150-\mathrm{W})$ power supply and an extra cooling fan support the custom WG circuit board which plugs into the PC backplane and draws $4 \mathrm{~A}$ from the $+5 \mathrm{~V}$ PC power supply.

\section{Safety Features}

The following features protect the subject from electrical injury even with multiple system faults:

Redundancy: Fig. 12 shows that at least three faults are required to connect the output to a dangerous voltage (the $\pm 120 \mathrm{~V} \mathrm{dc}$ supply). A combination of any two faults (for example, shorted $Q_{7}$ and shorted $C_{3}$ ) will allow a maximum of $1.2 \mathrm{~mA}$ to flow into the electrode. While 1.2 $\mathrm{mA} \mathrm{dc}$ is painful, it is not dangerous.

Power-Line Protection: The subject return electrode is connected directly to the equipment ground. Therefore, a ground-fault circuit interrupter (GFCI) in the $120 \mathrm{~V} \mathrm{ac}$ power line to the entire experimental apparatus protects the subject from receiving a dangerous shock if a major power-distribution fault occurs. The GFCI will also disconnect the ac power in the unlikely event that the subject touches a $120 \mathrm{~V}$ ac conductor in the ETSS.

Physical Layout: Circuitry with an electrical path to the subject is oriented or mounted so that broken or abraded wires, bent mountings, etc. are not able to contact dangerous voltages $(120 \mathrm{~V}$ ac or $\pm 120 \mathrm{~V} \mathrm{dc})$.

Footswitch: A footswitch which interrupts the $\pm 120 \mathrm{~V}$ dc supply allows the subject to quickly turn off the stimulation if the stimulation intensity becomes painfully high due to software failure.

Charge-Limiting Output Coupling: The value of the output coupling capacitors ensures that insufficient charge $(24 \mu \mathrm{C})$ can flow into a single electrode to cause ventricular fibrillation ( $560 \mu \mathrm{C}$-see below) in the subject, even with software failure or two circuit failures.

Consider the worst-case scenario in which $C_{4}$ in Fig. 12 is shorted and $C_{3}$ is initially charged with the circuit terminal $+120 \mathrm{~V}$ with respect to the load. Then suppose that $Q_{8}$ shorts, temporarily placing $-120 \mathrm{~V}$ on the electrode load. Current will flow through the load (subject) to reverse the polarity of charge on $C_{3}$. The required charge is $q=C v=(0.1 \mu \mathrm{F})(240 \mathrm{~V})=24 \mu \mathrm{C}$. This is the maximal charge that can flow into the subject with two system faults.

We arrive at the fibrillation charge of $560 \mu \mathrm{C}$ as follows. The accepted lower limit of ventricular fibrillation for whole-body, arm-to-arm $60-\mathrm{Hz}$ current flow in humans is $75 \mathrm{~mA}$ rms [17]. Roy et al. [18] show that electrical stimulation (extrasystole) of isolated rabbit hearts (using large electrodes to simulate whole-body current flow) occurs within the first half-cycle of ac stimulation at $500 \mathrm{~Hz}$. We assume that this is also true at $60 \mathrm{~Hz}$, because the period is longer and because the time constant for stimulation of the human myocardium is $0.6-1.1 \mathrm{~ms}$ [19]. Furthermore, the required average pulse current for external defibrillation of dog hearts is independent of pulse shape from 2-20 ms [20]. Therefore, the required human 
fibrillation charge in the first half-cycle of $75-\mathrm{mA} 60-\mathrm{Hz}$ stimulation is $(68 \mathrm{~mA}$ ave $)(8.3 \mathrm{~ms})=560 \mu \mathrm{C}$.

Electrode Design: Finally, coaxial electrodes (an active center electrode surrounded by a grounded ring) restrict the current flow to the vicinity of the electrodes. To cause fibrillation, the ground lead would have to break and the subject would have to touch a grounded object to provide a current path through the heart, in addition to the faults discussed above.

\section{Performance}

Operational Limits: Due to the limited supply voltage of $\pm 120 \mathrm{~V}$, the output coupling circuit $\left(C_{3}, C_{4}, R_{7}, R_{8}\right)$ imposes limits on the maximal output current into various loads with various waveforms. These are hard limits; minimal waveform distortion occurs before the limits are reached.

For functionally-monophasic pulses driving a $0-\Omega$ load, the maximal available current $I_{\mathrm{MAX}}(\mathrm{mA})$ is approximately $( \pm 15 \%)$ (approximately $2 \mathrm{nF}$ capacitance in parallel with $1-20 \mathrm{k} \Omega$ resistance [15]).

Linearity: The transconductance of $10 \mathrm{~mA} / \mathrm{V}$ varies +1 to $-4 \%$ over the useful operating range of $1-50 \mathrm{~mA}$ $(+1$ to $-2 \%$ for $2-30 \mathrm{~mA}$ ). Since this nonlinearity is somewhat predictable with current, the software corrects the linearity to $\pm 2 \%$ over $1-50 \mathrm{~mA}$. Also, since the VIC gain for positive and negative phases is often different, the WG has a provision for trimming the amplitude of the negative phase.

Dynamics: With a rectangular pulse at the input, the output current into $100 \Omega$ or into an electrode settles to its final value in $<1 \mu$ s with a maximal overshoot of $2 \%$. The pulse flatness is $\pm 0.5 \%$ for a relatively long (1-ms) pulse.

Timing: All of the waveform parameters except current are derived from the $5 \mathrm{MHz}$ crystal and hence their accuracy is limited only by the resolution of discrete frequency division in the Am9513 timer circuits (Table I).

$$
I_{\mathrm{MAX}}=\frac{k[1+(a / W)][1+c \ln (F)][1-d(N P B-1) \ln (P R R)]}{b+(N P B-1) I B I \cdot F}
$$

where the interburst interval $I B I=T-\left(10^{-3}\right) N P B \cdot P-$ $\left(10^{-6}\right) \mathrm{W}$. Experimental determination of the empirical constants for a least-squares fit yields $k=0.98, a=4800$, $b=1.08, c=0.31$, and $d=0.0057$. The unit for $I B I$ is $\mathrm{s}$; the other waveform parameter units are defined in $\mathrm{Ta}$ ble I.

For balanced-biphasic pulses, the maximal current into $0 \Omega$ is approximately $( \pm 10 \%)$

$$
I_{\mathrm{MAX}}=\frac{100 C}{W}
$$

where $C=50 \mathrm{nF}$ (the equivalent of $C_{3}$ and $C_{4}$ ).

The exact analytical expressions for (10) and (11) are very complex. Equation (10) is an empirical model which applies over the physiologically useful range of stimulation waveforms. Equation (11) determines the current which will cause $100 \mathrm{~V}$ to appear across the $C_{3}, C_{4}$ network during the first phase of a balanced-biphasic pulse.

A typical electrode requires $25-60 \mathrm{~V}$ depending on the stimulation current [15]. This reduces the maximal output current of the circuit into an electrode to approximately $I_{\text {MAX }} / 2$. For the electrodes used in most of our studies (5.5-mm diameter) the drive capability of this circuit for balanced-biphasic pulses is sufficient to produce painful stimulation if desired. Some functionally monophasic pulse trains with $N P B>1$ are not capable of delivering very strong stimulations due to the delimiting in (10).

Output Impedance: An ideal current source has an infinite output impedance. This circuit has a primarily resistive output impedance of $2 \mathrm{M} \Omega$, which results in $<1 \%$ change in current over the range of electrode impedances

\section{SOFTWARE}

The entire ETSS is controlled by the IBM PC through its bus. A custom software package translates a user input file containing commands for all of the waveform parameters for each of the 16 output channels into hardware commands for 1) the WG timers (which control all parameters except current) and 2) the analog section (which controls simulation current). The PC bus interface uses $\mathrm{I} / \mathrm{O}$ hex addressed B00 - B1F for the waveform generator and $\mathrm{I} / \mathrm{O}$ hex addresses $220-22 \mathrm{~F}$ for the analog system.

The software performs the following functions:

1) Read the input file containing the experiment control commands.

2) Check the input file for errors.

3) Randomize the order of stimulus presentations if specified.

4) Provide the specified subject instructions and prompts

5) Present the stimuli to the subject as defined in the input file.

6) Record the subject's responses, relevant parameters, and system error and status messages.

7) Sort the recorded data in the same order as the input file.

8) Generate an output file with recorded data.

\section{Development Tools}

We wrote most of the source code in Borland (Scotts Valley, CA) Turbo $\mathrm{C}$ version 2.0 along with the Turbo 
Assembler 1.0 and Turbo Debugger 1.0. Several timecritical functions use 8086 assembly code. Compiled drives (Analog Devices model AC1527-A) simplify communication with the analog system.

\section{Software Features}

Command Language: The ETSS software conducts experiments written in the ETSS command file language. This language supports directives and operation codes that enable the experimenter to control every feature of the system.

The command file consists of a header section and a body section. The programmer must define all variables, mathematical functions, blocks of text, and output file format commands in the header section. The body contains the data and commands that produce stimulations. The body is divided into groups of trials which are futher divided into parts. In addition, each trial is assigned a design number. This structure allows the system to randomize the execution order of experiments as well as sort result files in a consistent fashion.

Waveform Parameter Control: Every waveform parameter except for Type and $D$ may be updated in realtime (during an experimental trial) via mathematical functions. ETSS functions accept from one to three arguments and support operators such as logarithms, exponentiation, powers, roots, multiplication, division, addition, and subtraction. Arguments may be a combination of analog inputs (e.g., knobs connected to potentiometers), time, user-defined variables, or constants. A range-checking routine reports out-of-range parameters to the experimenter and prevents subsequent system malfunctions.

The command language provides user-defined variables, waveform parameter soft-limits, and analog "jitter." A variable such as an experimentally determined sensation threshold may be defined and used in a later trial. For example, the stimulation current for one trial can be a function of an earlier subject response.

The operational limits of the waveform parameters may be limited via a software command; thus, the system programmer can set the minimal and maximal operating value of a parameter. Furthermore, the programmer may specify the scope of this feature to affect any particular trial or the entire file.

The optional analog jitter function adds a random number to any desired analog input to prevent the subject from memorizing a particular potentiometer position for a repeated response.

Subject-Experimenter Interface: The ETTS software supports numerous methods of acquiring data from a test subject and displaying information to the subject. The subject may either enter data from the keyboard or from an analog input (e.g., by adjusting a potentiometer). The system always prompts the user when data inputs are required. At the end of each trial, the subject's responses are logged to an output result file for further processing. Long experiments may be saved and resumed at a later time. A group of experimental trials may be repeated if the subject makes a procedural error. At any time, the experimenter can append a comment to the output file.

Error Detection and Trapping: Experiments may run in either the normal mode or the debug mode. Normal mode operation displays only the information necessary for the subject to interact with the system. The debug mode displays the above information as well as information useful to the system programmer when debugging a command file, such as a real-time display of waveform parameters.

The ETSS system supports extensive error trapping, generating over one hundred error messages for conditions like out-of-range parameters and errors in command file syntax.

\section{Executable Modules}

The ETSS software consists of three executable files: 1) the command file preparser and randomizer, 2) the command file parser and interpreter, and 3) the result file organizer. An intelligent batch file sequentially invokes each file, aborting the process when necessary.

Preparser: If the experimenter specifies a randomized experiment, the command file preparser randomizes the execution order of the specified part of an experiment and creates a temporary reordered command file.

Parser and Interpreter: The command file parser and interpreter executes command files line by line. To optimize system performance, each part in the command file body is compiled prior to execution.

In addition, the system translates mathematical functions into Intel 8087 coprocessor machine code. Mathematical translation is accomplished as follows. First the function is formatted and operators are distinguished from variables and constants. Then the mathematical function is converted from infix to postfix notation. The conversion is necessitated by the 8087 coprocessor stack-based structure. Finally, each operator is translated into a sequence of Intel 8087 machine-language instructions.

Result File Organizer: The result file organizer sorts and formats the output files generated by the parser and interpreter and sorts the output file according to the design numbers associated with each trial. Thus, data from a randomized experiment are logged to a result file in a convenient order for analysis.

\section{Unusual Software Solutions}

IBM-PC Timer Interrupt: The ETSS system utilizes the IBM PC TIMER interrupt (interrupt zero) to conduct many of its time-dependent functions. The ETSS software changes the timer frequency to $20.0 \mathrm{~Hz}$ by reprogramming the 8253-5 programmable interval timer and installs a new interrupt service routine for the duration of the experiment. The original interrupt routine which updates the IBM PC system clock is not called by the new interrupt service routine, so upon exiting the system, the system clock must be reset from the hardware time-of-day clock. 
During the parsing of command file lines, the TIMER interrupt is disabled by masking the appropriate bit of the $8259 \mathrm{~A}$ programmable interrupt controller. The $\mathrm{PC}$ will function as expected with the TIMER interrupt disabled except when calling some DOS interrupt functions, for example, subfunction $08 \mathrm{H}$ of the DOS interrupt $21 \mathrm{H}$ (retrieve a character from the keyboard).

Real-Time Update of Am9513A Registers: The ETSS software updates the Am9513A system timing controllers in real-time. This may be accomplished without losing waveform integrity as long as one does not write to a counter during its terminal count. (During terminal count, the counter reloads itself from internal registers.) To avoid this situation, updating is synchronized with the 9513 oscillator via its FOUT (frequency. out) pin. The ETSS software configures the FOUT pin to produce a $50-\mathrm{kHz}$ signal derived by frequency division of the master oscillator. The software updates registers only after a level transition of FOUT to ensure terminal count does not occur during the update process (which lasts approximately $8 \mu \mathrm{s}$ ).

Complete schematic diagrams and software are available by contacting the third author.

\section{CONCLUSIONS}

The system described above is fully operational; it rapidly and automatically delivers a wide variety of electrotactile stimuli to subjects. Using this flexibility, we hope to determine 1) which waveforms can maximize dynamic range and comfort, 2) minimize error in identification of waveform parameter changes for information display, and 3) minimize skin irritation.

The ability to rapidly record large amounts of data has already yielded an interesting observation on the time variations in the electrotactile sensation threshold [21]. Ongoing experiments [22] include 1) the determination of how accurately a subject can control hand grasp force using electrotactile force feedback, 2) maximization of the perceived intensity of stimulation without discomfort, and 3) characterization of sensory adaptation to electrotactile stimulation.

\section{REFERENCES}

[1] K. A. Kaczmarek, J. G. Webster, P. Bach-y-Rita and W. J. Tompkins, "Electrotactile and vibrotactile displays for sensory substitution," IEEE Trans. Biomed. Eng., vol. 38, pp. 1-16, Jan. 1991.

[2] A. Y. J. Szeto and F. Saunders, "Electrocutaneous stimulation for sensory communication in rehabilitation engineering." IEEE Trans. Biomed. Eng., vol. BME-29, pp. 300-308, 1982.

[3] P. Bach-y-Rita, Brain Mechanisms in Sensory Substitution. New York: Academic, 1972

[4] C. C. Collins, "On mobility aids for the blind," in Electronic Spatial Sensing for the Blind, D. H. Warren and E. R. Strelow, Eds. Dordrecht. The Netherlands: Matinus Nijhoff, 1985, pp. 3564.

[5] C. M. Reed, N. I. Durlach, and L. D. Bradia, "Research on tactile communication of speech: A review," AHSA Monographs, vol. 20. pp. $1-23,1982$.

[6] C. E. Sherrick, "Basic and applied research on tactile aids for deaf people: Progress and prospects," J. Acoust. Soc. Amer., vol. 75, pp. $1325-1342,1984$.

[7] A. Y. J. Szeto and K. M. Christensen, "Technological devices for deaf-blind children: Needs and potential impact," IEEE Eng. Med. Biol. Mag., vol. 7, no. 3, pp. 25-29, 1988

[8] C. C. Collins and J. M. J. Madey, "Tactile sensory replacement," in Proc. San Diego Biomed. Symp., vol. 13, pp. 15-26, 1974.

[9] C. A. Phillips, "Sensory feedback control of upper- and lower-extremity motor prostheses." CRC Crit. Rev. Biomed. Eng., vol. 16 , pp. 105-140. 1988.

[10] R. R. Riso, "Sensory augmentation for enhanced control of FNS systems," in Ergonomics in Rehabilitation, A. Mital, Ed. New York: Taylor and Francis, 1988, pp. 253-271.

[11] F. A. Saunders, "Information transmission across the skin: Highresolution tactile sensory aids for the deaf and the blind," Intern. $J$. Neurosci, vol. 19, pp. 21-28, 1983

[12] A. Y. J. Szeto and R. R. Riso, "Sensory feedback using electrical stimulation of the tactile sense," in Rehabilitation Engineering, R. V. Smith and J. H. Leslie, Jr., Eds. Boca Raton, FL: CRC Press, 1990, pp. $29-78$

[13] F. A. Saunders, "Electrocutaneous displays," in Proc. Conf. Cutaneous Commun. Syst. Devices, pp. 20-26, 1973.

[14] A. S. Sedra and K. C. Smith, Microelectronic Circuits, 2nd ed. New York: CBS College, 1987.

[15] K. A. Kaczmarek and J. G. Webster. "Voltage-current characteristics of the electrotactile skin-electrode interface," in Proc. Annu. Int. Conf. IEEE Eng. Med. Biol. Soc., vol. 11, pp. 1526-1527, 1989.

[16] A. v. Boxtel, "Skin resistance during square-wave electrical pulses of 1 to $10 \mathrm{~mA}$," Med. Biol. Eng. Comput., vol. 15, pp. 679-687, 1977.

[17] W. H. Olsen, "Electrical safety." in Medical Instrumentation: Application and Design, J. G. Webster, Ed. Boston. Houghton Mifflin. 1978, pp. $667-707$

[18] O. Z. Roy, A. J. Mortimer, B. J. Trollope, and E. J. Villeneuve, "Effects of short-duration transients on cardiac rhythm," Med. Biol. Eng. Comput., vol. 22, pp. 225-228, 1984.

[19] J. A. Pearce. J. D. Bourland, W. Nielsen, L. A. Geddes, and M. Voelz. "Myocardial stimulation with ultrashort duration current pulses," $P A C E$, vol. 5, pp. 52-58, 1982.

[20] C. F. Babbs and J. D. Bourland, "Defibrillators," in Therapeutic Medical Devices: Application and Design, A. M. Cook and J. G. Webster, Eds. Englewood Cliffs, NJ: Prentice-Hall, 1982, pp. 6685.

[21] K. A. Kaczmarek, J. G. Webster, and R. G. Radwin, "Periodic variations in the electrotactile sensation threshold," in Proc. Annu. Int. Conf. IEEE Eng. Med. Biol. Soc., vol. 12, pp. 1060-1061, 1990.

[22] K. A. Kaczmarek, "Optimal electrotactile stimulation waveforms for human information display," Ph.D. thesis, Dep. Elec. Eng., Univ. Wisconsin-Madison, 1991.

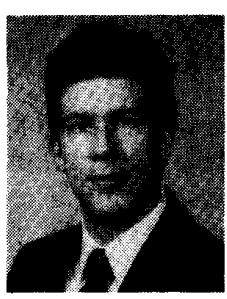

Kurt A. Kaczmarek ( $S^{\prime} 82-M^{\prime} 82-S^{`} 83-M^{`} 83-$ S'86) received the B.S.E.E. degree from the University of Illinois. Urbana, in 1982 and the M.S.E.E. degree from the University of Wisconsin, Madison, in 1984. In Madison he developed a vision-replacement system which sequentially presents image segments from a digital camera to a blind user via a vibrotactile display (Kaczmarek et al., IEEE Trans. Biomed. Eng., vol. BME-32, pp. 602-608, 1985).

From 1984 to 1986 he developed advanced medical product sterilization techniques at Baxter International. Round Lake, IL. He is currently working toward the Ph.D. degree in electrical engineering at the University of Wisconsin. Madison. His research will optimize the stimulation parameters for electrotactile display systems. These results will aid in development of a tactile feedback system for the insensate hand. He is a contributing author to Interfacing Sensors to the IBM $P C$, W. J. Tompkins and J. G. Webster, Eds. (Englewood Cliffs, NJ: Prentice-Hall, 1988). His interest areas include sensory substitution. somatosensory physiology. medical instrumentation, and physiological modeling. 


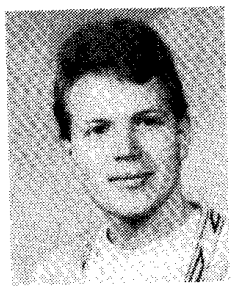

Kevin M. Kramer $\left(\mathrm{S}^{\circ} 89\right)$ received the B.S.E.E. degree from the University of Wisconsin, Madison, in 1991. where he is currently working toward the M.S.E.E. degree.

In Madison he works part-time at Persoft, Inc. developing local area network software applications. His interest areas include microelectronics, biomagnetism, and computer-based data-acquisition systems.

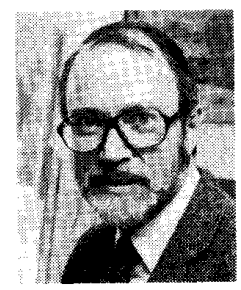

John G. Webster (M'59-SM'69-F'86) received the B.E.E. degree from Cornell University, Ith aca, NY, in 1953, and the M.S.E.E. and Ph.D. degrees from the University of Rochester, Rochester, NY, in 1965 and 1967, respectively.

$\mathrm{He}$ is Professor of Electrical and Computer Engineering at the University of Wisconsin-Madison. In the field of medical instrumentation he teaches undergraduate, graduate, and short courses, and does research on electrical impedance tomography, tactile sensors, electrodes, and biopotential amplifiers. He is author of Transducers and Sensors, An IEEE EAB Individual Learning Program (Piscataway, NJ: IEEE. 1989). He is coauthor, with B. Jacobson, of Medicine and Clinical Engineering (Englewood Cliffs, NJ: Prentice-Hall, 1977), and with R. Pallás-Areny, of Sen sors and Signal Conditioning (New York: Wiley, 1991). He is editor of Medical Instrumentation: Application and Design. 2nd ed. (Boston: Houghton-Mifflin, 1992), Encyclopedia of Medical Devices and Instrumentation (New York: Wiley, 1988), Tactile Sensors for Robotics and Medicine (New York: Wiley, 1988), Electrical Impedance Tomograph (Bristol, England: Adam Hilger, 1990), Teaching Design in Electrical Engineering (Piscataway, NJ: Educational Activities Board, IEEE, 1990). and Prevention of Pressure Sores: Engineering and Clinical Aspects (Bristol, England: Adam Hilger, 1991). He is coeditor, with A. M. Cook, of
Clinical Engineering: Principles and Practices (Englewood Cliffs, NJ: Prentice-Hall, 1979) and Therapeutic Medical Devices: Application and Design (Englewood Cliffs, NJ: Prentice-Hall, 1982), with W. J. Tompkins, of Design of Microcomputer-Based Medical Instrumentation (Englewood Cliffs, NJ: Prentice-Hall, 1981) and Interfacing Sensors to the IBM $P C$ (Englewood Cliffs, NJ: Prentice Hall, 1988), and with A. M. Cook, W. J. Tompkins, and G. C. Vanderheiden, of Electronic Devices for Rehabilitation (London: Chapman \& Hall. 1985).

Dr. Webster has been a member of the IEEE-EMBS Administrative Committee and the NIH Surgery and Bioengineering Study Section. He has been Associate Editor, Medical Instrumentation, of the IEEE Transactions on Biomedical Engineering, and Chairman of the IEEE-EMBS Fellow Committee.

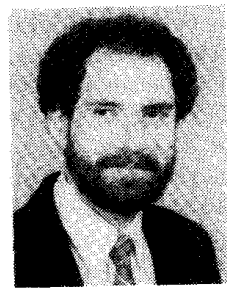

Robert G. Radwin ( $\mathrm{S}^{\prime} 84-\mathrm{M}^{\prime} 85$ ) received the B.S.E. degree in electrical engineering from the Polytechnic Institute of New York, Brooklyn, NY. in 1975 , M.S. degrees in electrical engineering and bioengineering in 1979. and the Ph.D. degree in industrial and operations engineering in 1986 all from The University of Michigan, Ann Arbor MI.

He was appointed as a Research Fellow at the Center for Ergonomics, Ann Arbor, Ml in 1986. He is currently an Assistant Professor at the University of Wisconsin-Madison in the Department of Industrial Engineering where he conducts research and teaches in the areas of ergonomics, human factors engineering, occupational health, and safety engineering. He is actively studying the causes and prevention of cumulative and repetitive trauma disorders in manual work, human performance measurement and instrumentation, occupational vibration exposure, and rehabilitation engineering.

Dr. Radwin received the Presidential Young Investigator Award in 1991. $\mathrm{He}$ is a member of Alpha Pi Mu, Eta Kappa Nu, and Sigma Xi. 